\title{
Cassini observations of plasmoid structure and dynamics: Implications for the role of magnetic reconnection in magnetospheric circulation at Saturn
}

\author{
C. M. Jackman, ${ }^{1}$ J. A. Slavin, ${ }^{2}$ and S. W. H. Cowley ${ }^{3}$ \\ Received 23 March 2011; revised 31 May 2011; accepted 28 June 2011; published 14 October 2011.
}

[1] We survey the Cassini magnetometer data during the deep tail orbits in 2006, and find 34 direct encounters with plasmoids. They occur as single, isolated events but also in groups of two or more plasmoids as is frequently observed at Earth. We show a case study example of three such plasmoids over three hours, where we estimate an upper limit of $5.68 \mathrm{GWb}$ of flux closure, and derive a reconnection rate over this interval of $526 \mathrm{kV}$. We show the results of a superposed epoch analysis of all 34 plasmoids indicating that, on average, plasmoids at Saturn are $\sim 8$ min in duration and they tend toward a loop-like, as opposed to flux rope-like topology, with little or no axial core magnetic field. Our analysis shows that plasmoids at Saturn are followed by an extended interval of the post-plasmoid plasma sheet (PPPS) lasting $\sim 58 \mathrm{~min}$. The average open magnetic flux disconnected by the continued reconnection following plasmoid formation that creates the PPPS is $\sim 3 \mathrm{GWb}$. We calculate expected recurrence rates for plasmoids, and compare these with a derived observational recurrence rate of one plasmoid every $\sim 2.4$ days, explaining the reasons why the spacecraft has not observed as many plasmoids as we predict will be released. We conclude that the Cassini magnetometer measurements require a combination of Vasyliunas-type closed-flux plasma sheet and Dungey-type open-flux lobe reconnection to account for the observed properties of the plasmoids and PPPS in Saturn's magnetotail.

Citation: Jackman, C. M., J. A. Slavin, and S. W. H. Cowley (2011), Cassini observations of plasmoid structure and dynamics: Implications for the role of magnetic reconnection in magnetospheric circulation at Saturn, J. Geophys. Res., 116, A10212, doi:10.1029/2011JA016682.

\section{Introduction}

[2] Since the arrival of the Cassini spacecraft at Saturn in July 2004, it has been possible to study the dynamics of the kronian magnetotail, and in particular, to search for in situ evidence of magnetic reconnection. The circulation of plasma within Saturn's magnetosphere is known to be largely driven by the planet's intense magnetic field, rapid rotation rate, and the transfer of mass to the magnetosphere by atmospheric outflow and the many satellite sources, in particular Enceladus. Nevertheless, solar wind-driven plasma circulation can also have an important influence, particularly in the outer magnetosphere [e.g., Badman and Cowley, 2007], and the role of magnetic reconnection is just beginning to be revealed by the Cassini measurements.

[3] The first evidence of tail reconnection was found on the outbound pass of the Saturn Orbit Insertion (SOI) maneuver in 2004, when Bunce et al. [2005] combined magnetometer,

\footnotetext{
${ }^{1}$ Department of Physics and Astronomy, University College London, London, UK.

${ }^{2}$ NASA Goddard Space Flight Center, Greenbelt, Maryland, USA.

${ }^{3}$ Department of Physics and Astronomy, University of Leicester, Leicester, UK.

Copyright 2011 by the American Geophysical Union. 0148-0227/11/2011JA016682
}

plasma and radio wave data and reported a compressiondriven tail reconnection and hot plasma injection event. In 2006 the spacecraft began a series of orbits exploring the deep magnetotail (out to $\sim 68 \mathrm{R}_{\mathrm{S}}$ ) in the equatorial plane. Such orbits were ideal for searching for the in situ evidence of magnetic reconnection in the cross-tail current sheet. On examination of data from these orbits, Jackman et al. [2007, 2008] and Hill et al. [2008] reported several observations of plasmoids and traveling compression regions (TCRs).

[4] While these in situ observations have given us more of an insight than ever before into the nature of reconnection in Saturn's magnetotail, our knowledge is still at a relatively early stage, particularly in comparison to the wealth of study that has been undertaken in the terrestrial magnetotail [e.g., Ieda et al., 1998; Slavin et al., 2003]. Nonetheless, there are already a number of sophisticated models of Saturn's magnetosphere which each provide insights into the global role of magnetic reconnection. For example, Zieger et al. [2010] studied mass release on Saturn's nightside through the application of a global MHD model. They found that under high and medium solar wind dynamic pressures, mass loss from the magnetotail is continuous and periodic respectively. For medium and low solar wind dynamic pressure, the model yields plasmoid repetition rates of $\sim 20-70 \mathrm{~h}$. The period gets shorter if an enhanced Dungey cycle is applied as an input, and vice versa if a larger axial tilt is assumed. Their model 
suggested that plasmoids generally have helical flux ropelike topology. Kidder et al. [2009] present a multifluid model which again yields plasmoids at Saturn. In this model the reconnection site(s) is in the region of $\sim 30 \mathrm{R}_{\mathrm{S}}$, formed preferentially in the post-midnight sector and plasmoids are released every few days. Jia et al. [2010] use global MHD simulations to investigate large-scale plasmoid formation in the tail and its effects on magnetospheric dynamics. They study how the process of plasmoid release is affected by the external solar wind conditions and by mass-loading from internal plasma sources. The characteristics of their model output plasmoids are: enhanced density and pressure, bipolar north-south field component, plus an enhanced field magnitude after the reconnection event. Fast plasma flows following plasmoid passage are interpreted as being associated with lobe reconnection which we discuss later. Many of the plasmoids released have a flux rope-like field line configuration. They find that after a shock-compression of the magnetosphere, reconnection in the magnetotail can be periodic on the timescale of the planetary rotation rate. However, such periodic behavior only lasts for a few cycles and thereafter the repetition time of plasmoid formation tends to become longer as the solar wind pressure gradually decreases.

[5] One of the advantages of global models is the ability to see where reconnection begins. The model outputs of Jia et al. [2010] yield a typical $\mathrm{x}$-line position at $\sim 40 \mathrm{R}_{\mathrm{S}}$ in the midnight to dawn sector. Following a shock-compression by the solar wind, they find that the $x$-line may move to $\sim 25 R_{S}$ while the width of the $\mathrm{x}$-line decreases. Such behavior is similar to that observed in the terrestrial magnetotail, where increases in solar wind velocity result in reconnection occurring closer to the Earth [e.g., Imber et al., 2011].

[6] What is the role of reconnection in the transport of plasma and magnetic flux at Saturn? While the rate is not well known observationally, open flux may be added to the magnetosphere through reconnection at the dayside, and this flux must ultimately be removed on the nightside, as part of the circulation pattern first described in the terrestrial context by Dungey [1961]. One way of ascertaining flux closure rates is through auroral monitoring. Saturn's main auroral oval emission is thought to be generated by upward-directed field-aligned current at the boundary between open and closed field lines [e.g., Cowley et al., 2004; Belenkaya et al., 2010], and thus heavily influenced by solar wind interaction. Using images from the Hubble Space Telescope (HST), Badman et al. [2005] assumed the poleward edge of Saturn's auroral emission to match the location of the open-closed field line boundary. From this they calculated the amount of flux contained within Saturn's polar cap. The value varied between $\sim 15-50 \mathrm{GWb}$. Because the amount of magnetic flux contained in the polar cap changes only in response to imbalances in the rates of reconnection at the magnetopause and in the cross-tail current sheet these results suggest that the dimensions of the magnetotail and its magnetic fields vary in a dynamic manner in response to Saturn's interaction with the solar wind. The timescale for the Dungey cycle at Saturn is estimated at $\sim 6$ to 10 days [e.g., Jackman et al., 2004; Badman and Cowley, 2007], obtained from using the estimated size of the open flux region to calculate the speed of anti-sunward flow on open field lines in Saturn's ionosphere, and hence the transit time across the open region. This timescale is thus comparable with the interval between cor- otating interaction region-related shock compressions of the magnetosphere [Jackman et al., 2004]. In this paper, we use Cassini magnetic field measurements collected in 2006 to determine the physical properties of plasmoids and the post-plasmoid plasma sheet (PPPS) at Saturn with a view to better understanding the role of magnetic reconnection in flux transport.

\section{Data Set and Event Selection}

[7] As mentioned above, data from the Cassini magnetometer [Dougherty et al., 2004] have been used to search for plasmoids in Saturn's magnetotail, as evidence of nightside reconnection [Jackman et al., 2007, 2008]. Plasmoids may be identified by a variation in the north/south field component, and, depending upon their topology, either a minimum in field magnitude for magnetic loops, or a maximum in the case of flux ropes [e.g., Hones et al., 1984; Moldwin and Hughes, 1992]. The best coordinate system to use when looking to identify such features in the data is Kronocentric Radial Theta Phi (KRTP), a spherical polar system referenced to the northern spin and magnetic axis of the planet. The radial component $\left(\mathrm{B}_{\mathrm{r}}\right)$ is positive outward from Saturn, the theta component $\left(\mathrm{B}_{\theta}\right)$ is positive southward, and the azimuthal component $\left(\mathrm{B}_{\varphi}\right)$ is positive in the direction of corotation (in a prograde direction). While changes in the north-south component can be indicative of reconnection $\mathrm{x}$-lines and their products, such as dipolarizations planetward of the reconnection site and plasmoids and fast tailward flow in the PPPS, the radial and azimuthal components can also be used to provide information on the position of the spacecraft relative to the central current sheet, and to elucidate the degree of corotation of the plasma (i.e., whether we are observing lagging or leading field lines). Jackman et al. [2009] illustrated the advantages of such a coordinate system, and emphasized the difference between unambiguously identifiable reconnection-related signatures and periodic field and plasma signatures in the tail associated with current sheet motion.

\section{Results}

\subsection{Case Study Example: 2006 Day 64}

[8] Figure 1 shows the interval from $08: 00-12: 00$ on day 64. The panels display the field in KRTP coordinates, as defined above. This interval encompasses three plasmoids which are marked with vertical dashed lines. Each plasmoid is indentified by a slow southward followed by a clear northward turning of the field accompanied by a decrease in the field magnitude indicating the presence of high plasma beta (the ratio of thermal to magnetic pressure). The beginning and end of the plasmoids are identified on the basis of the positive and negative extrema in $\mathrm{B}_{\theta}$ with the central event time roughly in the middle of the field deflection. The magnitude of the positive and negative (southward and northward) extremes in $\mathrm{B}_{\theta}$ are, in general, not equal. Any tilting of the current sheet and/or tail as a whole will introduce offsets in the signature, the plasmoid may deviate significantly from being cylindrically symmetric due to its interaction with the surrounding tail, the plasmoid may have been observed in the act of forming and not be time stationary, and the spacecraft path rarely goes through its exact center [cf. Slavin et al., 


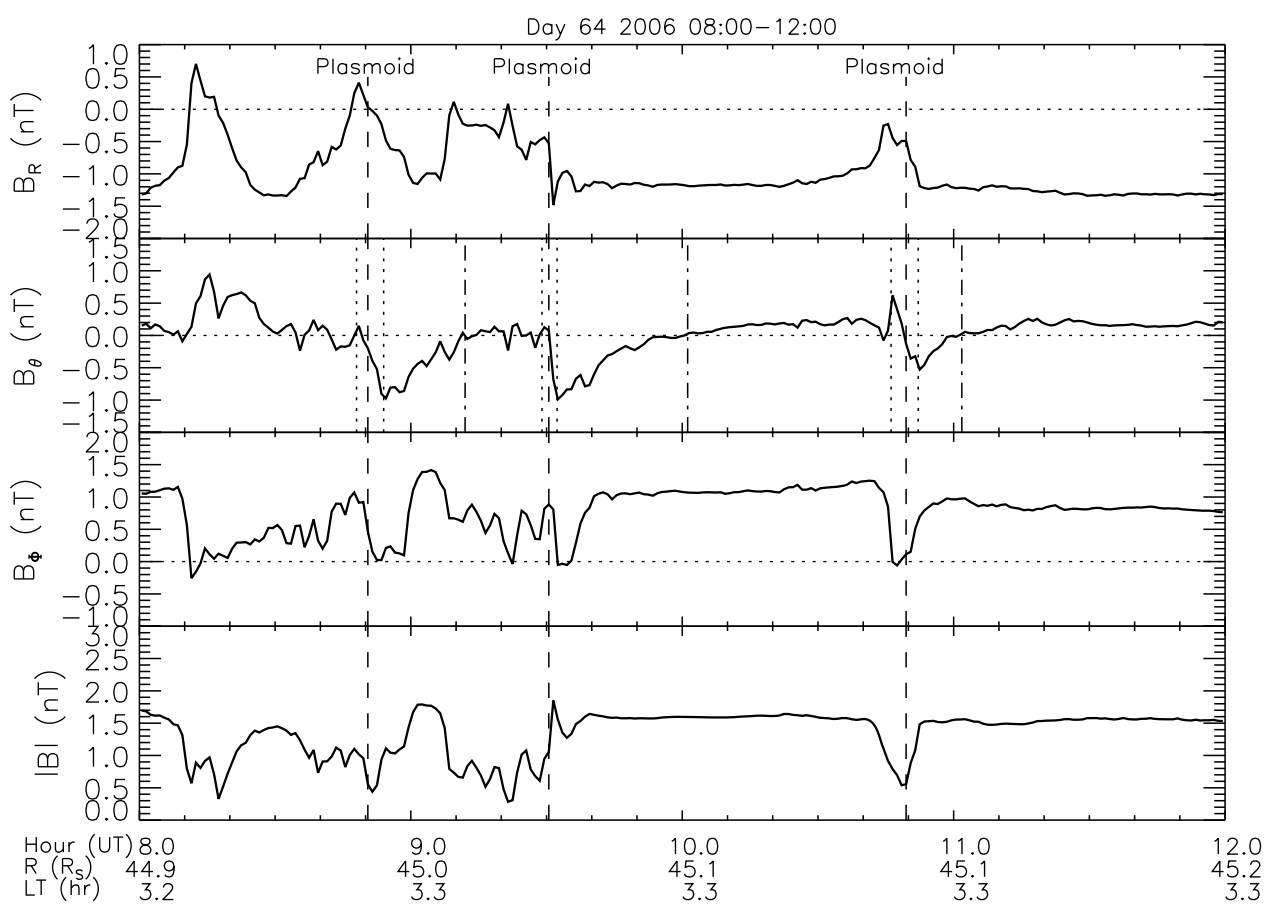

Figure 1. Magnetic field data in KRTP coordinates for 2006 day 64 08:00-12:00. Vertical dashed lines through all panels mark the central event time of three plasmoids. Vertical dotted lines on either side of each plasmoid mark the start and end times of the event based on a by-eye determination of the local extrema of the $\mathrm{B}_{\theta}$ component. Vertical dot-dashed lines mark the end of intervals of prolonged northward field, representing the PPPS. Time in hours, radial distance and local time of the spacecraft are given at the bottom of the plot.

2003]. The first plasmoid is observed at 08:51 with a total duration of $6 \mathrm{~min}$. There is then an extended interval of 18 min between the end of the plasmoid observation (marked by the most northward field) and the recovery of $\mathrm{B}_{\theta}$ to zero, the end of which is marked by a vertical dot-dashed line (as is the case for all three plasmoids shown here). This period of northward field is the PPPS [Richardson et al., 1987], where reconnection on open field lines from the lobes can proceed very rapidly, closing significant amounts of previously open flux. As we will discuss in more detail in section 4, the negative $\mathrm{B}_{\theta}$ in this interval is principally produced by the reconnection of open lobe field lines following the formation and ejection of the plasmoid. The second plasmoid at 09:31 is of a slightly shorter, i.e., $\sim 2 \mathrm{~min}$ duration, but in this case there is a $29 \mathrm{~min}$ interval of northward field after the end of the plasmoid. The third plasmoid is seen by the spacecraft at 10:49 for $6 \mathrm{~min}$, and the $\mathrm{B}_{\theta}$ component remains negative for $13 \mathrm{~min}$ after the event, $10 \mathrm{~min}$ of which correspond to the PPPS.

[9] The plasmoids in Figure 1 do not exhibit the strong core magnetic fields in the $\mathrm{B}_{\varphi}$ direction found in flux ropes [cf. Moldwin and Hughes, 1992; Slavin et al., 2003]. Instead they appear to have more loop-like topologies. For both types of plasmoid, the terrestrial analogy suggests that the primary magnetic flux transport is not due to the ejection of the plasmoid, but rather to the fast flow out of the $x$-line(s) after the plasmoid is ejected. It is the reconnection of the open lobe field lines after the plasmoid has formed that produces the fast sunward flow and transport of newly closed magnetic flux back toward the planet and the equally strong anti-sunward flow and transport of newly disconnected, interplanetary field lines down tail right behind the plasmoid, i.e., the PPPS [e.g., Richardson et al., 1987].

[10] The magnetic flux transported tailward in the PPPS per unit distance across the tail can be readily computed:

$$
\Phi_{\mathrm{PPPS}} / \mathrm{Ly}=\int \mathrm{V}_{\mathrm{PPPS}} \mathrm{B}_{\theta} \mathrm{dt}
$$

where $V_{\text {PPPS }}$ is the anti-sunward flow speed in the PPPS and the integration limits span the PPPS. $\Phi_{\text {PPPS }}$ is the total flux contained within the PPPS, and Ly is its width across the tail; i.e., essentially the width of the reconnection region. We must make some assumptions for the value of $\mathrm{V}_{\text {PPPS }}$, as unfortunately, due to spacecraft pointing constraints, it was not possible for us to obtain reliable in situ plasma velocity measurements for these three events. However, Hill et al. [2008] were successful in deriving velocities for two other plasmoids observed by Cassini in 2006, and thus based on their work it is reasonable to assume an average velocity of $800 \mathrm{~km} / \mathrm{s}$ tailward for plasmoids. This average value also agrees well with observations of plasmoids in the terrestrial tail [e.g., Ieda et al., 1998]. We note at this point that the tailward flow velocity in the PPPS may not be the same as that in the plasmoid itself. Theory indicates that closure of open field lines will result in rapid tailward flows within the plasma sheet comparable with the Alfvén speed in the lobes [e.g., Owen and Cowley, 1987], which can be in excess of $4000 \mathrm{~km} / \mathrm{s}$ at Saturn [Arridge et al., 2009]. Nonetheless, for the purposes of the calculations in this paper, we use the 

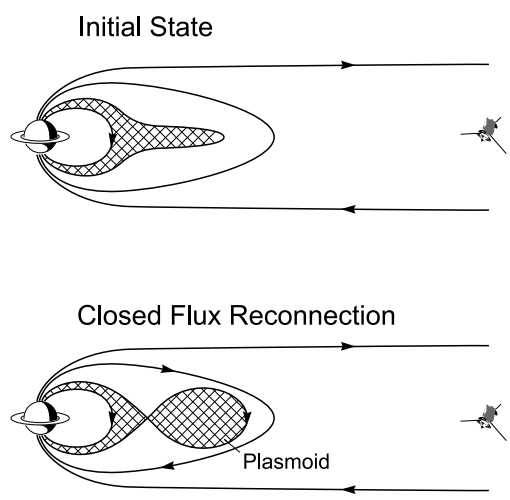

Closed \& Open Flux Reconnection

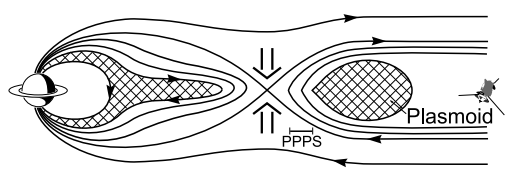

Disk Plasma
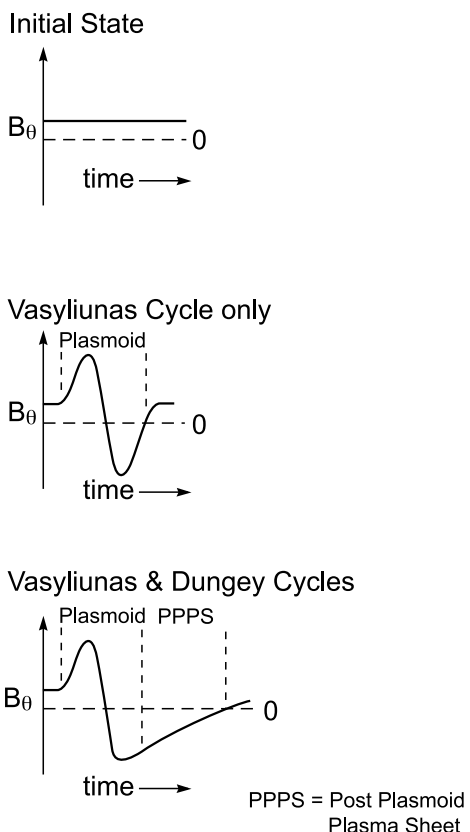

Figure 2. Schematic of three possible states of the magnetotail and the associated $\mathrm{B}_{\theta}$ signatures as measured by a spacecraft situated down the tail.

observed plasmoid flow velocity of $800 \mathrm{~km} / \mathrm{s}$, while bearing in mind that this may well be an under-estimate for the PPPS flows.

[11] Using the above method we integrated to obtain the magnetic flux closed via magnetic reconnection in the PPPS for each of the three cases shown here. Following the first plasmoid, the $\mathrm{B}_{\theta}$ component remained negative for $18 \mathrm{~min}$, and the flux closed is estimated at $0.470 \mathrm{~Wb} / \mathrm{m}$ across the tail. For the second, the field remained northward for $29 \mathrm{~min}$, with a total of $0.484 \mathrm{~Wb} / \mathrm{m}$ of flux transport estimated. Last, the third, shorter duration plasmoid was followed by a PPPS observation which lasted for $10 \mathrm{~min}$, and the flux closure estimate here is $0.094 \mathrm{~Wb} / \mathrm{m}$. Thus in the space of $\sim 3 \mathrm{~h}$, $1.048 \mathrm{~Wb} / \mathrm{m}$ of previously open magnetic flux was closed as a direct result of magnetic reconnection. We then note that if these reconnection regions occupied a significant fraction of the tail width as suggested by the modeling results cited in section 1, the open flux closed during the events would be significant. The full tail width at radial distances of $\sim 45 R_{S}$ where these plasmoids were observed is $\sim 90 \mathrm{R}_{\mathrm{S}}$ [e.g., Macek et al., 1992]. If for example, the plasmoids occupied this maximum width, the total open flux closure over this interval would be $\sim 5.68 \mathrm{GWb}$ (for a flow speed of $\sim 800 \mathrm{~km} / \mathrm{s}$ ), compared with typical open flux content in Saturn's tail of $\sim 40 \mathrm{GWb}$ deduced from auroral data [Badman et al., 2005].

\subsection{Magnetotail Reconnection and Magnetic Flux Closure}

[12] Figure 2 shows a schematic of various states of the magnetotail at Saturn. The top panel represents a state where no magnetotail reconnection is occurring. Jackman and Arridge [2011] noted that the average, or "steadystate" condition is for the $\mathrm{B}_{\theta}$ component to be small and positive (southward), and this is what would be expected for a spacecraft sampling field lines which are stretched out of a dipolar shape (which happens beyond $\sim 15 \mathrm{R}_{\mathrm{S}}$ at Saturn
[Arridge et al., 2007]). The second panel shows the situation where the Vasyliunas cycle is at work. As first described by Vasyliunas [1983] in a Jovian context, mass-loaded closed field lines rotate toward midnight from dusk, extend into the tail and then pinch off to form tailward-moving plasmoids. This release of plasmoids down the tail carries away the plasma added to the closed flux tubes in the forward magnetosphere. However, the Vasyliunas cycle does not include magnetic reconnection at the dayside magnetopause and, hence, it cannot involve open lobe flux tube reconnection. If this Vasyliunas-type closed field reconnection is the only type of reconnection taking place, then a spacecraft farther downstream would observe no net magnetic flux transport. In this case, the field signature which would be expected upon reconnection and plasmoid release is shown on the right-hand side of the middle panel. A spacecraft tailward of the reconnection point might see a bipolar signature in the $\mathrm{B}_{\theta}$ component: a small southward turning followed by a clear northward turning and then a return to small positive values. We note that reconnection of this type (i.e., on closed field lines) does not result in the removal of open flux from the magnetosphere.

[13] In the third panel we show the field line configuration and the expected magnetic field signature that would result from a combination of closed and open-field line reconnection. As mentioned in the introduction, the solar wind may drive cyclical flow in the magnetosphere, beginning with reconnection at the dayside magnetopause producing open flux tubes that are then transported by the solar wind flow to the magnetotail. The closure of these open flux tubes on the nightside via reconnection and their subsequent return to the dayside completes this cycle of transport known as the Dungey cycle. Badman and Cowley [2007] discussed the relative roles of the Dungey and Vasyliunas-cycles at Saturn and found that solar wind-controlled magnetospheric flows can play a major role under conditions of strong upstream 


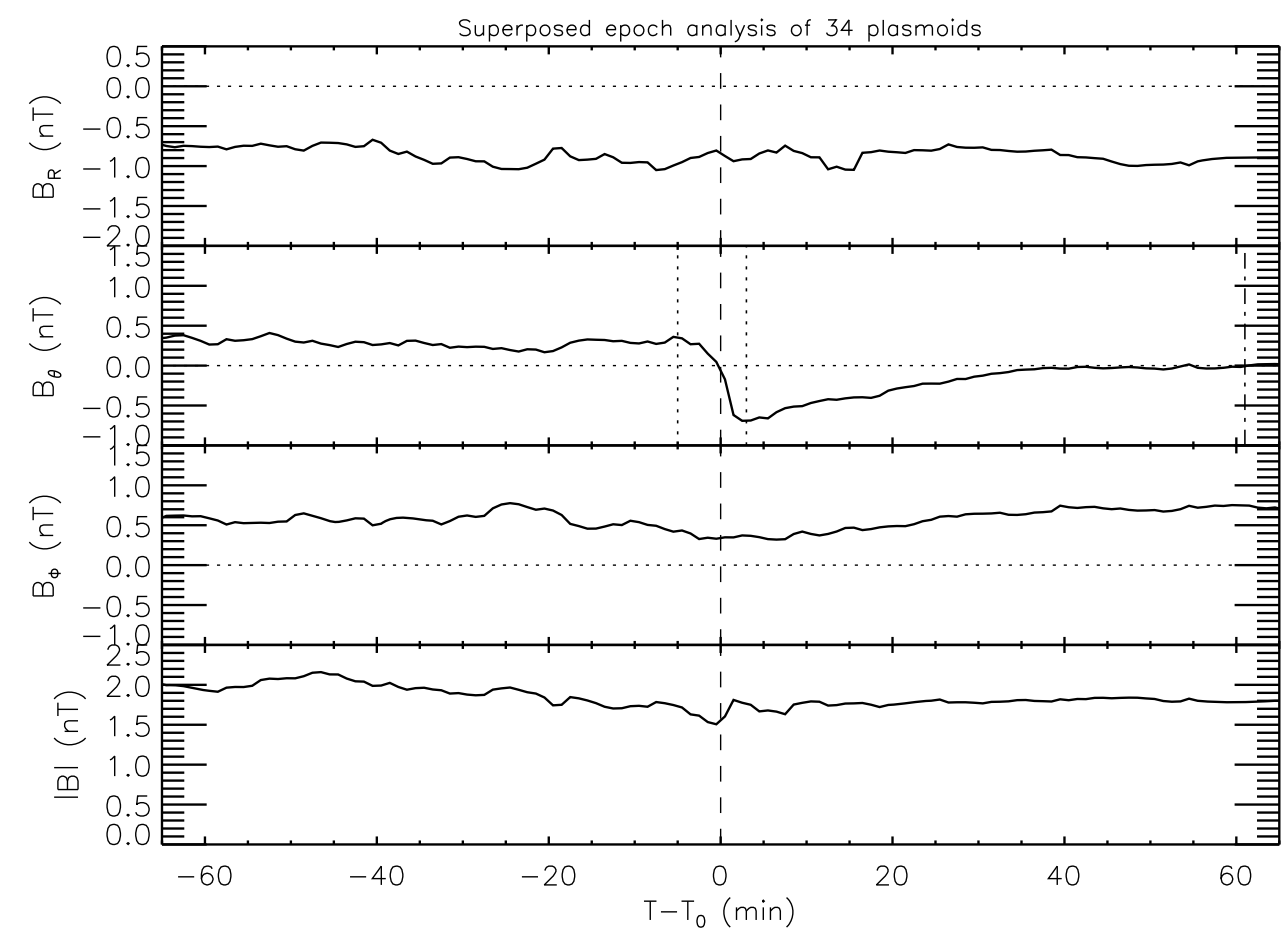

Figure 3. The radial, north-south and azimuthal components of the magnetic field together with the magnetic field strength for a superposed epoch analysis of 34 plasmoids at Saturn. The zero epoch is the central event time (marked by the vertical dashed line) and the plot range spans 65 min either side of the events. Vertical dotted and dot-dashed lines mark the start/end times of the average plasmoid and the end of the PPPS, in the same format as Figure 1.

driving. The expected field signature where both closed and open-field line reconnection is ongoing is shown in the bottom right hand panel of Figure 2. Here the start of the signature is the same as for Vasyliunas-cycle reconnection only, but the effect of the additional open field line reconnection is to extend the interval for which the field remains northward. We make the analogy here with the terrestrial PPPS, as discussed in section 3.1 above [e.g., Richardson et al., 1987], and we explore the nature of this field signature further in section 4 below.

\subsection{Average Plasmoid Signatures}

[14] We identified 34 plasmoids in the Cassini magnetometer data during the interval 2006 day 18-291. Figure 3 depicts the results of a superposed epoch analysis for these 34 plasmoids, where the zero epoch is the central event time. These events are the result of a detailed re-survey of the Cassini magnetometer data from 2006, and these, together with a number of TCR observations, are discussed in detail in a new paper which focuses on the statistical properties of reconnection signatures at Saturn (C.M. Jackman et al., manuscript in preparation, 2011). The superposed epoch approach allows us to see the average duration of plasmoid observations and the average magnitude of the associated field deflection. It also allows us to observe the field "compression" ahead of the plasmoid, any loop versus helical (flux rope) topology, and a measure of how long reconnection continued after the plasmoid was released (i.e., the extent of the PPPS observed). This will help us to see if Saturn reconnection events display "loading" and "unloading" features indicative of the inflation of the magnetotail lobes with magnetic flux during the growth phase of terrestrial substorms, and the subsequent closure of flux following the expansion phase [e.g., Fairfield, 1986; Slavin et al., 1992].

[15] We note that the radial field component is on average negative, implying that the spacecraft was generally below the center of the current sheet when observing plasmoids. This is biased heavily by the fact that Cassini spent much of 2006's equatorial orbits below the nominal current sheet position due to the hinging upwards of the sheet under conditions of southern hemisphere summer [e.g., Arridge et al., 2008]. In the absence of multipoint measurements, all that can be said is that the average distance of the spacecraft from the current sheet center is less than the half-height of the plasmoid. There is no significant change in the azimuthal component coincident with plasmoid passage.

[16] As noted above, the $\mathrm{B}_{\theta}$ component is on average small and positive in Saturn's tail [Jackman and Arridge, 2011]. However, this can change dramatically when reconnection is ongoing as the field lines in the magnetotail undergo reconfiguration. The main feature of the superposed epoch analysis is the strong northward turning of the field, centered around the zero epoch time. Physically this represents the passage of a tailward-moving plasmoid over the spacecraft which is further from the planet than the reconnection site. The amplitude of the field deflection from the southernmost (local maximum) to the northernmost (local minimum) either side of the event is $\sim 1 \mathrm{nT}$. As noted above, the magnitude of the positive and negative extrema in $\mathrm{B}_{\theta}$ are in general not equal, and this is reflected in the superposed epoch result where the local maximum and minimum are $\sim 0.3$ and $-0.7 \mathrm{nT}$ respectively. We note that this total field deflection of $\sim 1 \mathrm{nT}$ 
represents the average of 34 events, and that we have not normalized the observations to any common radial distance for this analysis. The observations occur over a range from 28 to $68 \mathrm{R}_{\mathrm{S}}$, with most in the region of $40-50 \mathrm{R}_{\mathrm{S}}$. We also note that the spacecraft trajectories through each of the 34 plasmoids may be very different, with some examples just skimming the edge of the structure, and others going closer to the center. The average plasmoid duration $(8 \mathrm{~min})$ is indicated by the vertical dashed lines at these southernmost and northernmost extrema either side of the central epoch time. After the end of the plasmoid observation, the field remains northward for $\sim 58 \mathrm{~min}$. As discussed in sections 3.1 and 3.2 above, this represents the PPPS and during this interval, lobe reconnection is ongoing and open flux which had been stored in the magnetotail is closed. The total field magnitude has a local dip coincident with the central event time. This is similar to what is seen for terrestrial magnetotail reconnection, in cases where plasmoids have a loop-like structure with no strong core field. We see little convincing evidence for a clear "growth phase," or increase in the field magnitude over an extended period prior to the event. This is due to the fact that the likely timescale over which magnetic flux accumulates in the kronian tail is several days, and thus it would not be visible on the timescale of this plot [Jackman et al., 2004]. A case study example of solar wind-driven tail inflation and subsequent collapse via reconnection several days later is shown by Jackman et al. [2010] and we refer the reader to this paper for a discussion of the accumulation of flux in the tail and the release of such flux after reaching a critical threshold.

[17] In order to estimate how much flux is closed on average through magnetotail reconnection episodes at Saturn, we can use the same method of calculation as detailed in section 3.1 above. Assuming a flow velocity of $800 \mathrm{~km} / \mathrm{s}$ (likely an under-estimate), and for a field that remains northward for $58 \mathrm{~min}$ after plasmoid passage, we calculate that $0.555 \mathrm{~Wb} / \mathrm{m}$ of flux would be closed via the PPPS on average.

\section{Magnetic Flux Transport}

\subsection{Plasmoids and the Post-Plasmoid Plasma Sheet}

[18] The plasmoid events in this study are identified by a bipolar signature in the field: a region of southward field followed by an interval of northward field. In the case of most of the plasmoid examples found at Saturn, this interval of northward field is significantly extended, and we argue that this represents the PPPS, a region of previously open flux that has been closed. From the superposed epoch analysis presented in section 3.3 above, we calculated based on equation (1) that an average flux of $0.555 \mathrm{~Wb} / \mathrm{m}$ was transported tailward via the PPPS (assuming a velocity of $800 \mathrm{~km} / \mathrm{s}$ ).

[19] In order to show that the flux within the PPPS cannot simply represent pinched-off closed field lines, we compare this value with estimates of the flux per unit cross-tail width lying within the pre-existing plasma sheet, given by

$$
\Phi_{\mathrm{PS}} / \mathrm{Ly}=\mathrm{B}_{\mathrm{PS}} \times \mathrm{D} \approx \mathrm{B}_{\mathrm{L}} / 2 \times \mathrm{D}
$$

where $B_{L}$ is the lobe field strength adjacent to the reconnection region, and $\mathrm{D}$ is the plasma sheet half-thickness. Taking $\mathrm{B}_{\mathrm{L}} \sim 3.5 \mathrm{nT}$ at radial distances of $\sim 30-40 \mathrm{R}_{\mathrm{S}}$ where plasmoids may typically form [Jackman and Arridge, 2011], and D 1.5 R [e.g., Kellett et al., 2009; Arridge et al., 2011], we find $\Phi_{\mathrm{PS}} \approx 0.16 \mathrm{~Wb} / \mathrm{m}$. Thus, the closed flux in the pinchedoff plasma sheet is considerably less than the flux transported in the PPPS $(\sim 0.555 \mathrm{~Wb} / \mathrm{m})$ by a factor of $\sim 3.5$. Indeed, this factor would increase considerably if a plausibly larger value were taken for the velocity of the tailward flow in the PPPS. Either way, it is clear that most of the flux transport following reconnection is due to closure of open field lines via the PPPS.

[20] To follow on from this point, we can also briefly investigate how much of the duration of the northward field signature then represents the plasmoid itself, compared with the PPPS. If the flux per unit length corresponding to the plasmoid is $\Phi_{\mathrm{PS}} / \mathrm{Ly} \approx 0.16 \mathrm{~Wb} / \mathrm{m}$ as just estimated, then taking $\mathrm{B}_{\theta} \sim 0.7 \mathrm{nT}$ from Figure 3 and $\mathrm{V}_{\mathrm{PS}} \sim 800 \mathrm{~km} / \mathrm{s}$, we estimate a plasmoid duration from equation (1) of $\sim 4.8 \mathrm{~min}$, comparable to those indicated in Figures 1 and 3. This is only a small fraction of the total time for which the field remains northward after the initial deflection, $58 \mathrm{~min}$ on average from the superposed epoch study. Thus the majority of the interval of negative $\mathrm{B}_{\theta}$ in these events corresponds to disconnected open flux in the PPPS.

\subsection{Plasmoid Recurrence Rates}

[21] What is the implication of the above for the time between plasmoids? Suppose we observe plasmoids in the tail in a region of cross-tail width, Dy, where the averaged interval between events at any point is $T_{P}$. If the open flux closed in each event per unit cross-tail width is $\Phi_{\text {PPPS }} / \mathrm{Ly}$, estimated above, then the overall averaged open flux closure rate due to these events is:

$$
\mathrm{R}_{\mathrm{C}}=\left(\Phi_{\mathrm{PPPS}} / \mathrm{Ly}\right) \times \mathrm{Dy} \times\left(1 / \mathrm{T}_{\mathrm{P}}\right) .
$$

Rearranging equation (3), we can thus determine the average time between each event at a given location to be

$$
\mathrm{T}_{\mathrm{P}}=\left(\Phi_{\mathrm{PPPS}} / \mathrm{Ly}\right) \times \mathrm{Dy} \times\left(1 / \mathrm{R}_{\mathrm{C}}\right) \geq\left(\Phi_{\mathrm{PPPS}} / \mathrm{Ly}\right) \times \mathrm{Dy} \times\left(1 / \mathrm{R}_{\mathrm{O}}\right)
$$

where $R_{O}$ is the average open flux production rate at the dayside magnetopause. The inequality follows from the fact that the open flux production rate $\mathrm{R}_{\mathrm{O}}$ must be larger than or equal to the plasmoid-related closure rate $\mathrm{R}_{\mathrm{C}}$, since some of the open flux may be closed by non-plasmoid-related reconnection, such as 'quiet time' reconnection in the more distant tail. As a starting point, let us take Dy to be the full tail width (corresponding to $\sim 90 \mathrm{R}_{\mathrm{S}}$ at radial distances of $\sim 45 \mathrm{R}_{\mathrm{S}}$ as mentioned in section 3.1 above). In addition, Jackman et al. [2004] used upstream interplanetary parameter measurements to estimate that the average rate of open flux production at Saturn is $\sim 45 \mathrm{kWb} / \mathrm{s}$, equivalent to a reconnection voltage of $45 \mathrm{kV}$, in good agreement with the in situ measurement of McAndrews et al. [2008]. Using these numbers in equation (4) together with $\left(\Phi_{\mathrm{PPPS}} / \mathrm{Ly}\right) \approx 0.56 \mathrm{~Wb} / \mathrm{m}$ from the superposed epoch analysis, we find a lower limit for $T_{P}$, the time between events, of $\sim 19 \mathrm{~h}$. This interval will then be larger if the PPPS flux is underestimated in the above calculation due to the assumption of a tailward plasma sheet speed that is too small. We also note that this estimate is completely independent of the cross-tail extent of each plasmoid (Ly). The smaller each plasmoid is, the more independent events have to occur across the tail so that the 
same rate of occurrence prevails at each point, as assumed. We address the question of the azimuthal extent of the reconnection region further in sections 4.3 and 4.4 below.

[22] It is then of interest to compare this estimate of the inter-event interval with our observations. To obtain a first order approximation to the observed recurrence rate of reconnection events in Saturn's tail, we have re-examined the data from 2006 day 18-291. We used a simple criterion to define the regions where plasmoids may be observed: nightside, beyond $20 \mathrm{R}_{\mathrm{S}}$, and close to the central plasma sheet $\left(\left|\mathrm{B}_{\mathrm{R}}\right|<1.5 \mathrm{nT}\right.$, where $\mathrm{B}_{\mathrm{R}}=0$ denotes the center of the current sheet). Cassini spends $\sim 82$ days in such regions and observes 34 plasmoids in this time. This suggests a recurrence rate of one plasmoid every $\sim 2.4$ days.

\subsection{Derived Reconnection Rates}

[23] Up until this point, we have derived the flux per unit distance across the tail. In order to obtain the total magnetic flux transformed from open lobe to closed or interplanetary magnetic flux, we must assume a realistic value for the azimuthal extent of the reconnection region, and below we take the full tail width of $90 \mathrm{R}_{\mathrm{S}}$ as our upper limit. The effects of changing this value are explored below in section 4.4. In the case study example shown in Figure 1, we estimate that $1.048 \mathrm{~Wb} / \mathrm{m}$ of previously open flux was closed as a direct result of magnetic reconnection over $\sim 3 \mathrm{~h}$. Assuming an azimuthal extent of $90 \mathrm{R}_{\mathrm{S}}$, the total flux closed in the three plasmoid-PPPS events is $5.68 \mathrm{GWb}$. Thus, with $1 \mathrm{kWb} / \mathrm{s}=$ $1 \mathrm{kV}, 5.68 \mathrm{GWb}$ over $3 \mathrm{~h}$ translates to a nightside reconnection rate of $\sim 526 \mathrm{kV}$. As noted by Badman and Cowley [2007], nightside reconnection rates during short, active intervals may be significantly higher than the average dayside reconnection rates. Jackman et al. [2004] employed an empirical formula adapted from Earth to calculate the expected reconnection voltage at Saturn's dayside magnetopause based on upstream solar wind parameters, and thus to estimate the amount of open flux added to the system over a given interval. They found that there may be extended ( $\sim 10$ day) intervals during corotating interaction region (CIR) rarefactions where reconnection rates are very low $(\sim 25 \mathrm{kV})$, and shorter ( $\sim 3$ day) intervals during CIR compressions where rates are much higher $(\sim 150 \mathrm{kV}$ and above), with an overall average reconnection voltage of $\sim 45 \mathrm{kV}$. Thus the reconnection rate of $526 \mathrm{kV}$ calculated over the interval in Figure 1 clearly corresponds to very active magnetotail conditions, even above those studied by Badman et al. [2005] in conjunction with a large auroral storm imaged by HST. In their paper, they noted that rarefaction regions typically lead to reconnection rates of $\sim 30-60 \mathrm{kV}$, while compression regions can result in rates of $\sim 100-200 \mathrm{kV}$.

[24] How many plasmoids will it take to the cycle the flux in the tail? From the superposed epoch analysis result in Figure 3, the flux transfer amount per plasmoid-PPPS event on average is: $0.555 \mathrm{~Wb} / \mathrm{m}$. Assuming reconnection across the full $90 \mathrm{R}_{\mathrm{S}}$ tail width, we obtain a value of $3.01 \mathrm{GWb}$ for the average flux closed per event. Since Saturn's magnetic tail contains about $40 \mathrm{GWb}$ of magnetic flux, it would take $\sim 13$ such plasmoid-PPPS events to cycle all of the flux in the tail. As mentioned in the introduction, the Dungey cycle time for Saturn is estimated at $\sim 6$ to 10 days [e.g., Jackman et al., 2004; Badman and Cowley, 2007]. Thus from the plasmoidPPPS results presented here, this suggests a mean plasmoid occurrence rate of $\sim 1.3-2.2$ plasmoids per day based on the estimate of $90 \mathrm{R}_{\mathrm{S}}$ azimuthal extent. The real rate could be much lower than this for a number of reasons. First, if the PPPS tailward speed is much higher than $800 \mathrm{~km} / \mathrm{s}$ (e.g., closer to $4000 \mathrm{~km} / \mathrm{s}$, the Alfvén speed in the lobes quoted above), then $\Phi_{\text {PPPS }} /$ Ly will be much larger than the estimate above, leading to a longer waiting time between successive plasmoids. Second, only part of the open flux added at the dayside may be closed through reconnection such as we have observed here. Rather, some might be closed via reconnection at a distant $\mathrm{x}$-line, or through smaller scale processes [Bagenal and Delamere, 2011]. Finally, further open flux could be closed after the reconnection site has retreated tailward of the spacecraft and our measurements miss this.

[25] As mentioned above, the rate of flux addition to the magnetosphere through reconnection at the dayside is intermittent due to the nature of the solar wind CIR pattern, and thus we might reasonably expect that flux closure on the nightside also occurs in concentrated bursts. It may be triggered externally by solar wind changes, or internally as the tail flux content exceeds some threshold for instability [e.g., Milan et al., 2005]. Badman and Cowley [2007] estimated tail reconnection rates of up to $450 \mathrm{kV}$, occurring for $\sim 1.5$ days per solar rotation. This is in reasonable agreement with our observations here, where during the interval shown in Figure 1, a short period of intense reconnection and flux closure in Saturn's tail was observed, with an estimated reconnection rate of $526 \mathrm{kV}$.

\subsection{Effects of Changing Azimuthal Extent of Reconnection Region}

[26] For all of the calculations above, we have assumed that reconnection can take place across the full width of the tail, and our current Cassini observations are not sufficient to support or indeed to refute this idea due to insufficient trajectory coverage. However, we can draw some analogy with the terrestrial magnetotail, where plasmoids are typically estimated to occupy up to two-thirds of the width of the tail on the basis of statistical studies [Ieda et al., 1998] and rare multispacecraft observations [Slavin et al., 1999]. This twothirds would correspond to a $60 \mathrm{R}_{\mathrm{S}}$ azimuthal extent of the reconnection region on Saturn's nightside. If we take this value instead of the full tail width of $90 R_{S}$ as we assumed for all the calculations above, it would yield the following results: an average of $\sim 2.01 \mathrm{GWb}$ of flux per event based on the superposed epoch analysis, leading to a recurrence rate of 2-3 plasmoids per day. It would also yield a reconnection rate of $350 \mathrm{kV}$ instead of the $526 \mathrm{kV}$ that we estimate. Future modeling work may help to constrain the size of the kronian reconnection region, while continued improvements in the analysis of plasma data from the Cassini spacecraft may enable us to improve our estimates of the velocity of flows in plasmoids and in the PPPS. For the time being, our calculations as presented here serve as a guide to both observational analysts and modellers as to the larger role that reconnection plays in the reconfiguration of Saturn's magnetosphere and the driving of global dynamics.

\section{Summary}

[27] We have shown using data from the Cassini magnetometer that plasmoids are observed in Saturn's magnetotail, 
not just in isolation, but also in groups, or "chains." Our interpretation of the case study shown here is that all three plasmoids are linked to a common reconnection event. As the first plasmoid is seen by the spacecraft at a radial distance of $\sim 45 \mathrm{R}_{\mathrm{S}}$, we conclude that the $\mathrm{x}$-line was planetward of this location. The deflection in the north-south field component is of similar magnitude for all three events. This combined with the fact that over the 4-h interval shown, the spacecraft position did not change substantially means that we may reasonably assume that all three plasmoids were of a similar height and azimuthal extent.

[28] We went on to present the field signatures that would be expected for reconnection on closed and open field lines. Our observations show that both the Dungey and Vasyliunas cycle regimes may be present and that magnetotail reconnection at Saturn can close a significant amount of the open flux that is known (through auroral observation) to accumulate in the lobes.

[29] Our results indicate that plasmoids at Saturn are quite similar in many respects to those seen at Earth, but with a longer temporal duration, and on average a structure that is less helical, or twisted, than at Earth. While the frequency of occurrence and east-west extent of plasmoids and tail reconnection $\mathrm{x}$-lines are still not well determined at Saturn, our calculations suggest that $\sim 1.3-2.2$ plasmoid ejections per day are required to match the estimated $\sim 6-10$ day Dungey cycle time scale. An approximate recurrence rate from observation is lower than this calculated value, but several possible explanations have been outlined for this. When plasmoid ejections do occur, we may expect them to happen in short, concentrated bursts. Future work will explore the triggers for magnetotail reconnection at Saturn and seek to further constrain the plasmoid recurrence rate observationally.

[30] Acknowledgments. C.M.J. would like to thank Neal Powell at Imperial for artwork. Work at Imperial College London was funded by the Science and Technology Facilities Council (STFC). We acknowledge M.K. Dougherty for provision of the Cassini magnetometer data and S. Kellock and the team at Imperial College London for MAG data processing. C.M.J. and J.A.S. acknowledge useful discussions with the International Space Science Institute (ISSI) "Dynamics of Planetary Magnetotails" team led by C.M.J. C.M.J. 's work at UCL is funded through a Leverhulme Trust Early Career Fellowship. Work at the University of Leicester was supported by STFC grant ST/H002480/1.

[31] Masaki Fujimoto thanks the reviewers for their assistance in evaluating this paper.

\section{References}

Arridge, C. S., C. T. Russell, K. K. Khurana, N. Achilleos, N. Andre', A. M. Rymer, M. K. Dougherty, and A. J. Coates (2007), Mass of Saturn's magnetodisc: Cassini observations, Geophys. Res. Lett., 34, L09108, doi:10.1029/2006GL028921.

Arridge, C. S., K. K. Khurana, C. T. Russell, D. J. Southwood, N. Achilleos, M. K. Dougherty, A. J. Coates, and H. K. Leinweber (2008), Warping of Saturn's magnetospheric and magnetotail current sheets, J. Geophys. Res., 113, A08217, doi:10.1029/2007JA012963.

Arridge, C. S., et al. (2009), Plasma electrons in Saturn's magnetotail: Structure, distribution and energisation, Planet. Space Sci., 57, 2032-2047, doi:10.1016/j.pss.2009.09.007.

Arridge, C. S., et al. (2011), Periodic motion of Saturn's nightside plasma sheet, J. Geophys. Res., doi:10.1029/2011JA016827, in press.

Badman, S. V., and S. W. H. Cowley (2007), Significance of Dungey cycle flows in Jupiter's and Saturn's magnetospheres, and their identification on closed equatorial field lines, Ann. Geophys., 25, 941-951, doi:10.5194/angeo-25-941-2007.
Badman, S. V., E. J. Bunce, J. T. Clarke, S. W. H. Cowley, J.-C. Gérard, D. Grodent, and S. E. Milan (2005), Open flux estimates in Saturn's magnetosphere during the January 2004 Cassini-HST campaign, and implications for reconnection rates, J. Geophys. Res., 110, A11216, doi:10.1029/2005JA011240.

Bagenal, F., and P. A. Delamere (2011), Flow of mass and energy in the magnetospheres of Jupiter and Saturn, J. Geophys. Res., 116, A05209, doi:10.1029/2010JA016294.

Belenkaya, E. S., I. I. Alexeev, M. S. Blokhina, E. J. Bunce, S. W. H. Cowley, J. D. Nichols, V. V. Kalegaev, V. G. Petrov, and G. Provan (2010), IMF dependence of Saturn's auroras: Modelling study of HST and Cassini data from 12-15 February 2008, Ann. Geophys., 28, 1559-1570, doi:10.5194/angeo-28-1559-2010.

Bunce, E. J., S. W. H. Cowley, D. M. Wright, A. J. Coates, M. K. Dougherty, N. Krupp, W. S. Kurth, and A. M. Rymer (2005), In situ observations of a solar wind compression-induced hot plasma injection in Saturn's tail, Geophys. Res. Lett., 32, L20S04, doi:10.1029/ 2005GL022888.

Cowley, S. W. H., E. J. Bunce, and J. M. O'Rourke (2004), A simple quantitative model of plasma flows and currents in Saturn's polar ionosphere, J. Geophys. Res., 109, A05212, doi:10.1029/2003JA010375.

Dougherty, M. K., et al. (2004), The Cassini magnetic field investigation, Space Sci. Rev., 114, 331-383, doi:10.1007/s11214-004-1432-2.

Dungey, J. W. (1961), Interplanetary magnetic field and the auroral zones, Phys. Rev. Lett., 6, 47-48, doi:10.1103/PhysRevLett.6.47.

Fairfield, D. H. (1986), Time variations of the distant magnetotail, Geophys. Res. Lett., 13(1), 80-83, doi:10.1029/GL013i001p00080.

Hill, T. W., et al. (2008), Plasmoids in Saturn's magnetotail, J. Geophys. Res., 113, A01214, doi:10.1029/2007JA012626.

Hones, E. W., Jr., D. N. Baker, S. J. Bame, W. C. Feldman, J. T. Gosling, D. J. McComas, R. D. Zwickl, J. A. Slavin, E. J. Smith, and B. T. Tsurutani (1984), Structure of the magnetotail at $220 \mathrm{R}_{\mathrm{E}}$ and its response to geomagnetic activity, Geophys. Res. Lett., 11, 5-7, doi:10.1029/ GL011i001p00005.

Ieda, A., S. Machida, T Mukai, Y Saito, T. Yamamoto, A. Nishida, T. Terasawa, and S. Kokubun (1998), Statistical analysis of plasmoid evolution with GEOTAIL observations, J. Geophys. Res., 103, 4453-4465, doi:10.1029/97JA03240.

Imber, S. M., J. A. Slavin, H. U. Auster, and V. Angelopoulos (2011), A THEMIS survey of flux ropes and traveling compression regions: Location of the near-Earth reconnection site during solar minimum, J. Geophys. Res., 116, A02201, doi:10.1029/2010JA016026.

Jackman, C. M., and C. S. Arridge (2011), Statistical properties of the magnetic field in the kronian magnetotail lobes and current sheet, J. Geophys. Res., 116, A05224, doi:10.1029/2010JA015973.

Jackman, C. M., N. Achilleos, E. J. Bunce, S. W. H. Cowley, M. K. Dougherty, G. H. Jones, S. E. Milan, and E. J. Smith (2004), Interplanetary magnetic field at $\sim 9 A U$ during the declining phase of the solar cycle and its implications for Saturn's magnetospheric dynamics, J. Geophys. Res., 109, A11203, doi:10.1029/2004JA010614

Jackman, C. M., C. T. Russell, D. J. Southwood, C. S. Arridge, N. Achilleos, and M. K. Dougherty (2007), Strong field dipolarizations in Saturn's magnetotail: In situ evidence of reconnection, Geophys. Res. Lett., 34, L11203, doi:10.1029/2007GL029764.

Jackman, C. M., et al. (2008), A multi-instrument view of tail reconnection at Saturn, J. Geophys. Res., 113, A11213, doi:10.1029/2008JA013592.

Jackman, C. M., C. S. Arridge, H. J. McAndrews, M. G. Henderson, and R. J. Wilson (2009), Northward field excursions in Saturn's magnetotail and their relationship to magnetospheric periodicities, Geophys. Res. Lett., 36, L16101, doi:10.1029/2009GL039149.

Jackman, C. M., C. S. Arridge, J. A. Slavin, S. E. Milan, L. Lamy, M. K. Dougherty, and A. J. Coates (2010), In situ observations of the effect of a solar wind compression on Saturn's magnetotail, J. Geophys. Res., 115, A10240, doi:10.1029/2010JA015312.

Jia, X., K. C. Hansen, T. I. Gombosi, M. G. Kivelson, G. Toth, D. De Zeeuw, and A. J. Ridley (2010), Global MHD simulation of the interaction between Saturn's magnetosphere and the solar wind, Abstract SM31C-01 presented at 2010 Fall Meeting, AGU, San Francisco, Calif., 13-17 Dec.

Kellett, S., E. J. Bunce, A. J. Coates, and S. W. H. Cowley (2009), Thickness of Saturn's ring current determined from north-south Cassini passes through the current layer, J. Geophys. Res., 114, A04209, doi:10.1029/2008JA013942.

Kidder, A., R. Winglee, and E. M. Harnett (2009), Multi-fluid simulations of plasmoid development at Saturn, Eos Trans. AGU, 90(52), Abstract SM31D-04.

Macek, W. M., W. S. Kurth, R. P. Lepping, and D. G. Sibeck (1992), Distant magnetotails of the outer magnetic planets, Adv. Space Res., 12(8), 47-55, doi:10.1016/0273-1177(92)90376-9. 
McAndrews, H. J., C. J. Owen, M. Thomsen, B. Lavraud, A. J. Coates, M. K. Dougherty, and D. T. Young (2008), Evidence for reconnection at Saturn's magnetopause, J. Geophys. Res., 113, A04210, doi:10.1029/2007JA012581.

Milan, S. E., E. J. Bunce, S. W. H. Cowley, and C. M. Jackman (2005), Implications of rapid planetary rotation for the Dungey magnetotail of Saturn, J. Geophys. Res., 110, A03209, doi:10.1029/2004JA010716.

Moldwin, M. B., and W. J. Hughes (1992), On the formation and evolution of plasmoids: A survey of the ISEE 3 data, J. Geophys. Res., 97, 19,259-19,282, doi:10.1029/92JA01598.

Owen, C. J., and S. W. H. Cowley (1987), Simple models of timedependent reconnection in a collision-free plasma with an application to substorms in the geomagnetic tail, Planet. Space Sci., 35, 451-466, doi:10.1016/0032-0633(87)90102-4

Richardson, I. G., S. W. H. Cowley, E. W. Hones Jr., and S. J. Bame (1987), Plasmoid-associated energetic ion bursts in the deep geomagnetic tail: Properties of plasmoids and the post-plasmoid plasma sheet, J. Geophys. Res., 92, 9997-10,013, doi:10.1029/JA092iA09p09997.

Slavin, J. A., M. F. Smith, E. L. Mazur, D. N. Baker, T. Iyemori, H. J. Singer, and E. W. Greenstadt (1992), ISEE 3 plasmoid and TCR observations during an extended interval of substorm activity, Geophys. Res. Lett., 19(8), 825-828, doi:10.1029/92GL00394.
Slavin, J. A., et al. (1999), Dual spacecraft observations of lobe magnetic field perturbations before, during and after plasmoid release, Geophys. Res. Lett., 26(19), 2897-2900, doi:10.1029/1999GL003606.

Slavin, J. A., R. P. Lepping, J. Gjerloev, D. H. Fairfield, M. Hesse, C. J. Owen, M. B. Moldwin, T. Nagai, A. Ieda, and T. Mukai (2003), Geotail observations of magnetic flux ropes in the plasma sheet, J. Geophys. Res., 108(A1), 1015, doi:10.1029/2002JA009557.

Vasyliunas, V. M. (1983), Plasma distribution and flow, in Physics of the Jovian Magnetosphere, edited by A. J. Dessler, pp. 395-453, Cambridge Univ. Press, New York, doi:10.1017/CBO9780511564574.013.

Zieger, B., K. C. Hansen, T. I. Gombosi, and D. L. De Zeeuw (2010), Periodic plasma escape from the mass-loaded Kronian magnetosphere, J. Geophys. Res., 115, A08208, doi:10.1029/2009JA014951.

S. W. H. Cowley, Department of Physics and Astronomy, University of Leicester, University Road, Leicester LE1 7RH, UK.

C. M. Jackman, Department of Physics and Astronomy, University College London, Gower Place, London WC1E 6BT, UK. (caitriona jackman@ucl.ac.uk)

J. A. Slavin, NASA Goddard Space Flight Center, Code 670, Greenbelt, MD 20771, USA. 